\title{
Interactive comment on "Three-dimensional distribution of fine particulate matter concentrations and synchronous meteorological data measured by an unmanned aerial vehicle (UAV) in Yangtze River Delta, China” by Si-Jia Lu et al.
}

Anonymous Referee \#2

Received and published: 13 April 2016

General The paper presents a setup and results of measurements of PM2.5 and BC concentrations and basic meteorological parameters by using an unmanned aerial vehicle (UAV) at a site in eastern China. With this setup the 3-dimensional distribution of pollutants can be measured which is undoubtedly very useful, for instance for evaluating the performance of air pollution forecasting models. In principle the work is ok and worth publishing but there are some shortcomings. 
the correct one for this work. My opinion is based on that as far as measurement methods, data processing, or algorithms or such are concerned, there are actually no new technical or methodological innovations or observations in this paper. Numerous aerosol measurements using UAVs have been published during the last few decades. So, as a concept there is nothing new. The instruments installed in the aircraft are all commercial, made by well known manufacturers. In the data processing there are essentially no new innovations either. However, as I wrote above, the results of this work are useful, for instance for evaluating the performance of air pollution forecasting models. Therefore a more suitable journal would be one that has the atmosphere itself as its focus, not methods as in AMT.

\section{Detailed comments}

P1,L25: "... Among all the air pollutants, the fine particulate matter is responsible for the climate change and..." This is not true. Particles do have radiative effects for sure, but mainly cooling. It is the greenhouse gases that are responsible for climate change. If all aerosol emissions were stopped, climate change would continue due to GHGs, probably faster.

The language should be corrected. As a non-native English speaker I don't want to be too picky, my English is not error free either. But in this text there are very many sentences and expressions that make it difficult to read and need correction. Here are just a few examples:

P1,L28: I would recommend changing the word "dissipation" to dispersion. Actually, the word "dissipation" is used in several sentences of the paper but in none of them it is really the good term.

P2,L1: " The only ground-based observations were not sufficient..." Rewrite as "Ground-based observations only are not sufficient..."

P2,L5-6 : " Studies ... were routinely conducted by meteorological tower..." This means 
that there was a meteorological tower who conducted studies. In other words, the expression "were conducted by" gives an impression of that the tower is a person. And the tense is misleading: "were conducted" suggests that they are not conducted any more.

P2,L8-9: " .. it is limited to monitoring elevation (no more than $350 \mathrm{~m}$ ) and mobility." This would mean something is monitoring elevation, in other words monitoring whether someting is rising or falling. And "monitoring mobility" would mean monitoring, whether something is moving. Rewrite.

A related note: the word "monitoring" is used throughout the text in an uncorrect way. In aerosol science monitoring generally means long-lasting, continuous measurements. For example at an air quality measurement station. The UAV measurements in this paper are not monitoring, unless the flights are more or less continuous, which they are not.

P2, L25: " unmanned aircraft vehicle" Should be "unmanned aerial vehicle"

\section{Section 2.2}

What is the manufacturer and model of the aircraft? What is its fuel.

Table 1. The aethalometer does not measure pressure. What did you use for measuring $p$ ?

P5.L2-4: Describe the results of the intercomparison better than giving just one correlation coefficient. How long was the intercomparison, show scatter plots, and regression lines, slope and offset..

Table 2 needs a proper, detailed caption and column explanations.

P6,L21 What does "self-monitoring" mean?

P7,L5. The formula (1) is not from Day et al. (2000), where is it from? They gave growth of scattering coefficient and it is not the same as the growth of mass. And it 
varies with the chemical composition. -what is the reasoning for using this CF? An it is unclear from the explanation, how did you use the CF. Did you correct with it all PM concentrations to $\mathrm{RH} 0 \%$ or what? Secondly, if I put in the formula any $\mathrm{RH}>2$ and 100 , the formula gives negative values. Correct it.

In the time series figures, add dates in the x-axes to make it easier to read.

Move section 3.4 earlier because the met data are used in the explanations of the profiles.

Table 5 is useless.

Interactive comment on Atmos. Meas. Tech. Discuss., doi:10.5194/amt-2016-57, 2016.

Interactive

comment \\ Discussion paper}

